\title{
Fiber Bragg grating measurement technology in industry
}

\author{
Markus J. Schmid ${ }^{1}$, Mathias S. Müller ${ }^{1}$, Alexander W. Koch ${ }^{1}$ \\ ${ }^{1}$ Institute for Measurement Systems and Sensor Technology, Technische Universität München, \\ Theresienstr. 90/N5, Munich 80333, Germany, \\ mar.schmid@tum.de
}

\begin{abstract}
:
Fiber Bragg grating (FBG) sensor technology offers new possibilities for monitoring and control applications through advantages as low influence by electro-magnetic interference, inherent lightning protection, and high durability. In this publication we discuss two concepts of fiber optic measurement systems and their characteristics regarding number of sensors, accuracy of measurement, real-time capability, temperature compensation, and costs. Furthermore we give examples of industry applications using fiber optic measurement technology.
\end{abstract}

Key words: fiber Bragg grating, sensor, measurement, industrial

\section{Introduction}

Fiber optic measurement technology offers potential for advanced sensor applications.

Properties as no electro-magnetic interference, no conductive material, light weight, small size, integration in composite materials, long distances due to low attenuation, low noise dependency through frequency modulation, cascading using wavelength multiplexing, and high strain capability are major advantages of this technology.

Following we discuss two concepts for fiber Bragg grating measurement systems. Furthermore we demonstrate industrial applications, where the two concepts use their individual potential best.

\section{Theory}

A fiber Bragg grating (FBG) is a periodic refractive index modulation in the core of an optical fiber. The modulation is transversally inscribed over a length of about 1 to $10 \mathrm{~mm}$. The grating can be seen as a narrow-band resonator, which responses with the characteristic Bragg reflection under broadband illumination (see Fig. 1). The central Bragg-wavelength

$$
\lambda_{B}=2 \bar{n}_{e} \Lambda
$$

depends on the effective refractive index $\bar{n}_{e}$ of the grating and its period length $\Lambda$.

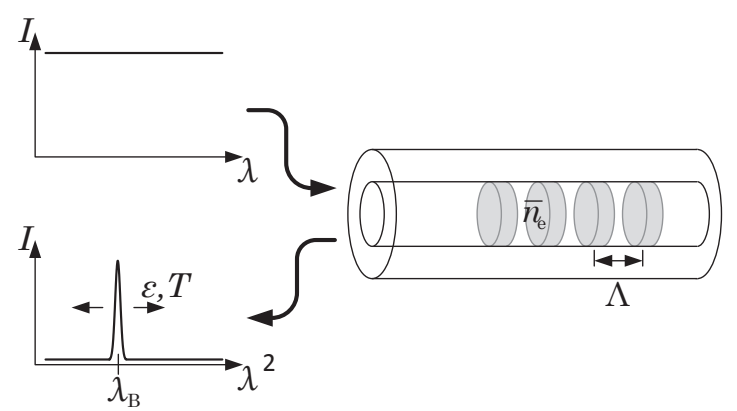

Fig.1.The fiber Bragg grating works as a narrowband mirror.

Fiber Bragg grating sensors are dominantly sensitive to temperature $T$ and axial strain $\varepsilon$ through $\bar{n}_{e}(T, \varepsilon)$ and $\Lambda(T, \varepsilon)$ [1]. Therefore a FBG system can measure physical values by a transducer converting the measurement quantity to a shift in Bragg-wavelength [2].

\section{Measurement technology}

Fiber optic measurement systems may be distinguished in two concepts - a multiplexing system and a single sensor system.

Multiplexing systems support multiple fiber Bragg grating sensors in a single fiber by using wavelength - (WDM), spatial - (SDM), or time division (TDM) concepts, or even a combination of these. Single sensor systems are only capable for one Bragg grating per fiber.

Following to the introduction of measurement systems we want to discuss their advantages and disadvantages in various categories. 


\section{Measurement systems}

The major components of a multiplexing FBG measurement system are the light source and the detector unit. Dependent on the functionality of these components the fiber optic measurement system supports wavelength - or time division multiplexing (see Fig. 2).

Wavelength division multiplexing is either realized by a tunable light source [3], or a dispersive element or a tunable filter in front of the photo detector [1, 4]. Time division multiplexing can be realized by a pulsed light source [5]. In [6] Luo et al. describe a combination of wavelength - and time division multiplexing.

Spatial division multiplexing is combinable with wavelength - and time division multiplexing and can be realized by an optical switch like in Fig. 2.

An exemplarily single sensor edge-filter based system can be seen in Fig. 3. The Bragg reflection of the sensor illuminates the two photodiodes, where one is filtered by an edgefilter [7, 8]. The following signal processing enables a nearly linear, continuous projection of the Bragg-wavelength - carrying the physical value - to the electrical measurement signal.

Number of sensors per fiber

A typically spectrometric or tunable light source measurement setup at $1550 \mathrm{~nm}$ has up to $100 \mathrm{~nm}$ spectral range. For a dynamic wavelength range of $5 \mathrm{~nm}$ per cascaded sensor, the system supports 20 FBGs per sensor fiber. Less dynamic wavelength range enlarges the number of sensors per string in favor of less potential for signal modulation.

A time division multiplexing system allows about 100 cascaded FBGs per sensor fiber dependent on the power of the light source and the reflectivity of the FBGs. The combination of time - and wavelength division multiplexing offers up to 2,000 sensors per fiber.

By an optical switch the number of sensors per measurement system can be multiplied by the factor of spatial states. Each port of the optical switch supports one sensor fiber. In favor of more sensors by spatial division multiplexing the sampling rate of each sensor decreases by the speed of the optical switch and the number of spatial states.

The edge-filter measurement system just supports one fiber Bragg grating per fiber. This concept is similar to conventional electrical strain sensors with a single measuring amplifier for each sensor. To support more FBGs the system needs to be parallelized. An optical switch is not suitable for edge-filter concepts, because of long response times of the switch.

Accuracy of measurement

WDM based systems offer a high accuracy of measurement especially with integrated optical etalons or gas cells. Cyclic self-calibration routines combined with depolarization can increase absolute measurement accuracy below $\pm 1 \mathrm{pm}$. For a dynamic sensor range of $5 \mathrm{~nm}$ this means a relative accuracy of measurement of $\pm 0.02 \%$. A standard C-band fiber Bragg grating with temperature sensitivity of $6.67 \mathrm{ppm} / \mathrm{K}$ therefore allows a resolution of $\pm 0.1 \mathrm{~K}$ over $500 \mathrm{~K}$.

A time division multiplexing system also supports internal references. Due to serial cascading at a fixed wavelength the sensorsignal is rather weak. The absolute measurement accuracy is about $\pm 10 \mathrm{pm}$. The described temperature measurement can be performed with a reduced resolution of $\pm 1 \mathrm{~K}$.

The concept of edge-filter systems excludes internal references. The total accuracy of measurement is about $\pm 10 \mathrm{pm}$. A typical dynamic sensor range of $10 \mathrm{~nm}$ leads to a relative accuracy of $\pm 0.1 \%$. A standard C-band fiber Bragg grating with axial strain sensitivity of $0.78 \mathrm{ppm} / \mathrm{\mu m} / \mathrm{m}$ therefore allows an accuracy of $\pm 8 \mu \mathrm{m} / \mathrm{m}$ over more than $8000 \mu \mathrm{m} / \mathrm{m}$.

\section{Anti-aliasing}

Some applications for monitoring and control require a measurement system with antialiasing filtering.

Multiplexing fiber Bragg grating interrogators discretize the time-signal in the optical domain without a possibility for analog low-pass filtering. Due to the Nyquist-Shannon sampling theorem aliasing effects cannot be avoided. Therefore unknown transient effects can cause misinterpretation of monitoring systems or even critical states in control loops.

Furthermore the complex computation of the sensor signals out of the optical spectrum leads to latency dependent on the calculating capacity. Hard real-time standards in industry applications can conflict with the system performance.

A typical measurement setting of spectrometer or tunable laser has $1 \mathrm{kHz}$ sampling frequency. Similar time division multiplexing systems have low sampling rates due to time interleaved measurements. Typical systems offer up to $100 \mathrm{~Hz}$ sampling rate per fiber Bragg grating. Such systems are well suited for quasi static measurements like distributed temperature measurement for process monitoring. 


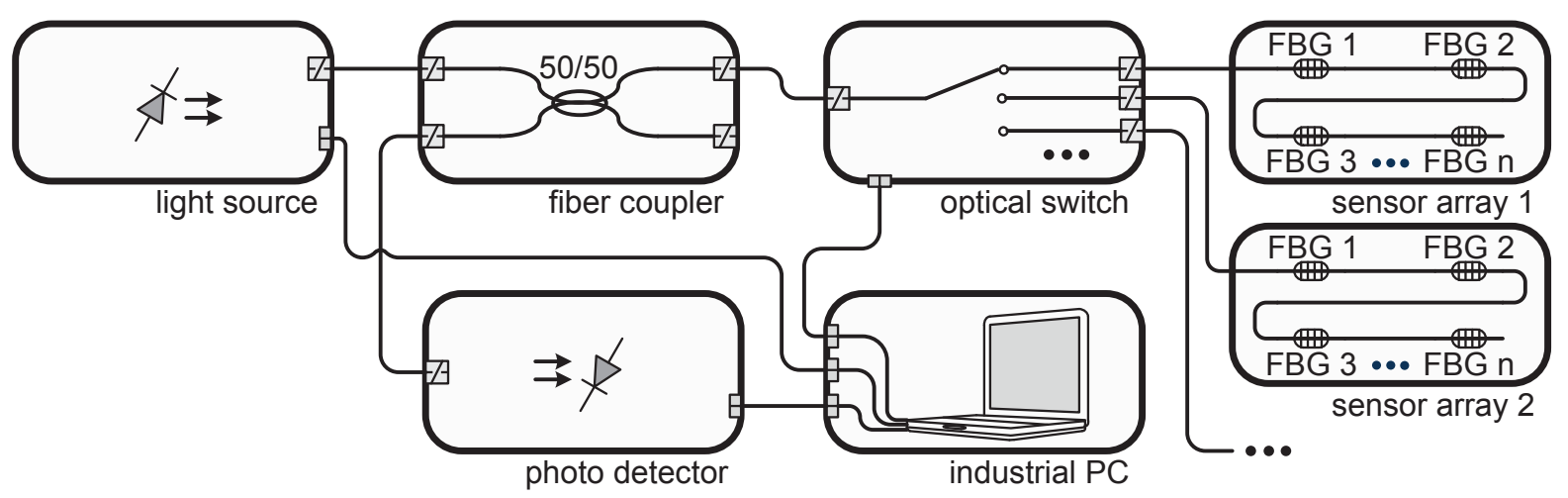

Fig.2. Multiplexing fiber Bragg grating measurement system using wavelength -, time-, spatial division multiplexing, or a combination of these. Wavelength division multiplexing is either realized by a tunable light source or a spectrometric photo detector. Time division multiplexing can be realized by a pulsed light source and the spatial division multiplexing by an optical switch.

Contrary to the multiplexing system the edgefilter concept is suitable for anti-aliasing filtering. The wavelength modulated sensor signal illuminates the two photodiodes continuously, where one is filtered by an edge-filter. The transmission of the edge-filter is nearly linear.

Therefore the ratio of the edge-filtered and the unfiltered intensity offers a linear, attenuationneutral transformation of the Bragg-wavelength to an analog electrical signal. Further this signal can be filtered with conventional signal processing devices suppressing aliasing effects.

Due to the linear sensor signal low computation capability is needed. This enables minor latency and high signal frequencies. The system structure allows sampling rates up to multiple $\mathrm{MHz}$.

\section{Temperature compensation}

Fiber Bragg grating sensors have a cross dependency in temperature and strain. For strain measurements temperature effects must be compensated. This includes the temperature expansion of the measurement object itself.

There are two approaches to temperature compensation for fiber Bragg grating measurement technology - passive and active temperature compensation.

Passive concepts use a mechanical strain transduction structure, which induces a contradirectional temperature dependent strain. Thereby the structure can compensate both the temperature induced strain shift of the measurement object and the temperature dependent sensor effect. This concept enables temperature compensated strain measurement with a single fiber Bragg grating. It can be used with edge-filter as well as multiplexing systems.

For the active temperature compensation a second mechanically decoupled thermally coupled fiber Bragg grating measures temperature effects, which can be compensated afterwards in signal processing. The quality of compensation is dependent on the thermal coupling between strain and temperature sensor. This concept is often used for multiplexing measurement systems.

\section{Costs}

The costs of the measurement system can be separated to fix costs of the interrogator and costs per sensor. We consider the costs of the measurement system not including the fiber Bragg grating sensor. The sensor is between 100 and 1,000 Euro per sensor dependent on its complexity. All discussed prices are of 2015 regarding vendors known to the authors (Micron Optics, FBGS, Sensuron, fos4X, Insensys/Moog, and Smart Fibers).

A typical wavelength division multiplexing system with 4 to 32 spatial states has fix costs of 15 to 50 thousand Euro. Thus the devices support between 80 and 640 sensors with costs per sensor around 80 to 200 Euro.

The time division multiplexing system is applicable for 100 to 50,000 fiber Bragg gratings. Fix costs of the system lie between 10 and 75 thousand Euro, and thus costs per sensor between two and 100 Euro.

The edge-filter measurement system with four to 16 sensor-channels has fix costs between five and 15 thousand Euro. Single sensor systems with reduced specifications have the potential for 500 to 1,000 Euro. Thus costs per sensor for edge-filter systems lie between one and two thousand Euro. 


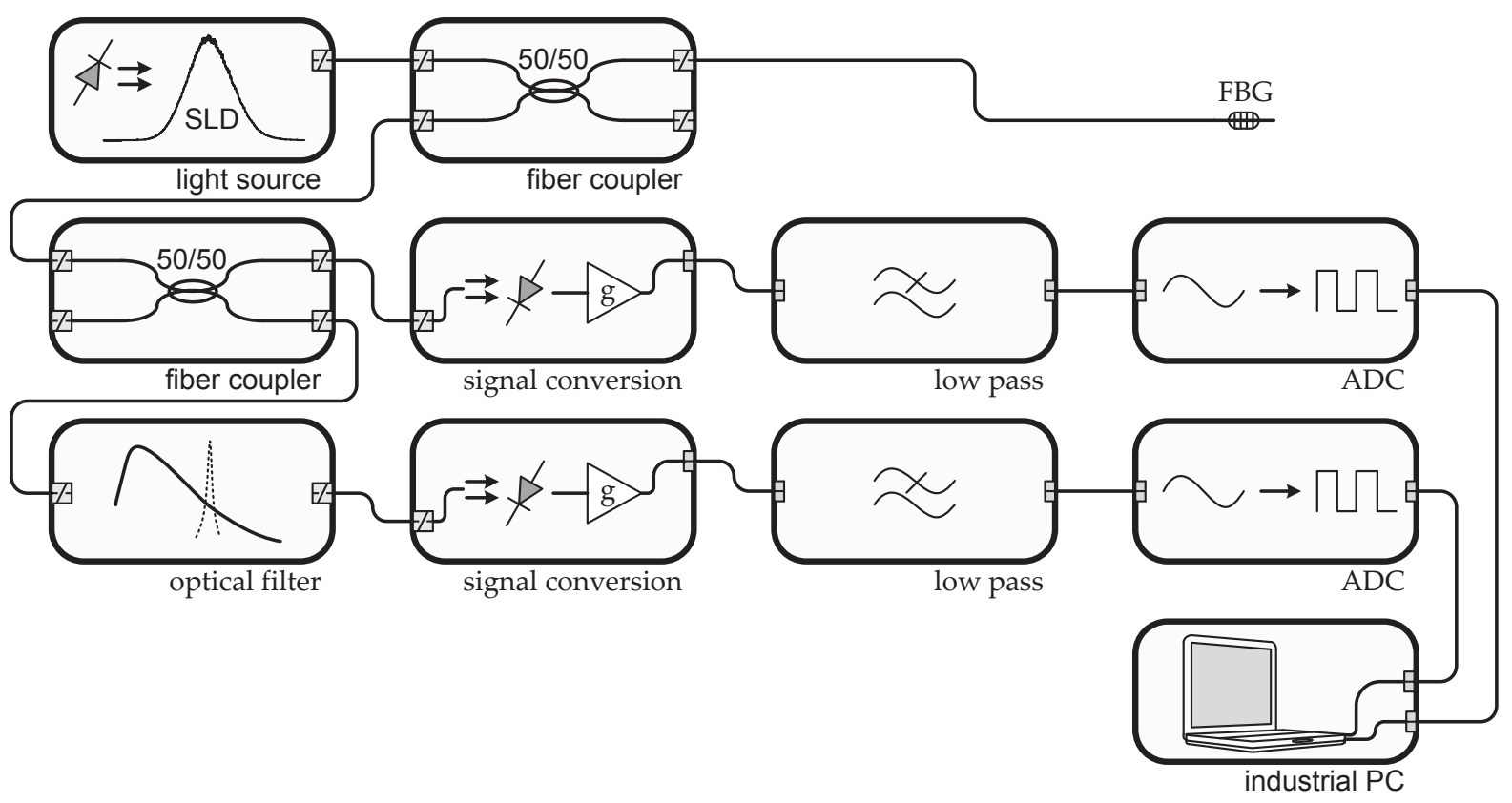

Fig.3. Edge-filter based fiber Bragg grating measurement system enabling analog low-pass filtering and therefore real anti-aliasing.

\section{Industry applications}

Following we discuss two industry applications using the individual advantages of multiplexing and single fiber optic measurement technologies.

Both technologies enable measurement applications where low electro-magnetic interference, high temperature stability, low wearout, and/or high strain amplitudes are required.

In chemical reactors temperature monitoring with around 100 to 500 sensors at sampling rates of 5 to $20 \mathrm{~Hz}$ is needed to control the reaction. This distributed temperature measurement is well covered by a wavelength - or time division multiplexing system.

In wind energy smart blades deliver a great potential to reduce loads induced through turbulent wind fields to future rotors with diameters beyond 150 meter. Active individual pitch control within sub-seconds therefore bases on a dynamic blade load measurement complying real-time and anti-aliasing requirements. Using fiber Bragg grating edgefilter measurement systems enables transient strain and acceleration monitoring of the rotor blades. 
Tab. 1: Comparison of WDM, TMD, and edge-filter measurement systems.

\begin{tabular}{|c|c|c|c|}
\hline & WDM system & TDM system & Edge-filter system \\
\hline Number of sensors & 1 to 620 & 1 to 50,000 & 1 to 16 \\
\hline $\begin{array}{l}\text { Accuracy of } \\
\text { measurement }\end{array}$ & $\pm 0.02 \%$ & $\pm 0.1 \%$ & $\pm 0.1 \%$ \\
\hline Anti-aliasing & $\begin{array}{c}\text { no, } \\
\text { sample rate }<1 \mathrm{kHz}\end{array}$ & $\begin{array}{c}\text { no, } \\
\text { sample rate }<100 \mathrm{~Hz}\end{array}$ & $\begin{array}{c}\text { yes, } \\
\text { sample rate }<1 \mathrm{MHz}\end{array}$ \\
\hline $\begin{array}{l}\text { Temperature } \\
\text { compensation }\end{array}$ & active and passive & active and passive & passive \\
\hline Costs & $\begin{array}{c}15 . .50 \mathrm{k} €(\mathrm{fix}) \\
80 . .200 €(\text { per sensor })\end{array}$ & $\begin{array}{c}10 . .75 \mathrm{k} €(\text { fix }) \\
2 . .100 €(\text { per sensor })\end{array}$ & $\begin{array}{c}2 . .15 \mathrm{k} €(\mathrm{fix}) \\
1 . .2 \mathrm{k} € \text { (per sensor) }\end{array}$ \\
\hline
\end{tabular}

\section{References}

[1] A. D. Kersey, et al, Fiber Grating Sensors, Journal of Lightwave Technology 15, 1442-1463 (1997); doi: 10.1109/50.618377

[2] M. Trutzel, Dehnungsermittlung mit faseroptischen Bragg-Gitter-Sensoren, Dissertation, Berlin (2001)

[3] M. Müller, et al, Fiber-Optic Sensor Interrogation Based on a Widely Tunable Monolithic Laser Diode, IEEE Transactions on Instrumentation and Measurement 15, 696-703 (2010); doi: 10.1109/TIM.2009.2025987

[4] A. D. Kersey, et al, Multiplexed fiber Bragg grating strain-sensor system with a fiber FabryPerot wavelength filter, Optics Letters 18, 13701372 (1993); doi: 10.1364/OL.18.001370

[5] D. J. F. Cooper, et al, Simple and Highly Sensitive Method for Wavelength Measurement of Low-Power Time-Multiplexed Signals Using Optical Amplifiers, Journal of Lightwave Technology 21, 1612-1620 (2003); doi: 10.1109/JLT.2003.814393

[6] Z. Luo, et al, A time- and wavelength-division multiplexing sensor network with ultra-weak fiber Bragg gratings, Optics Express 21, 22799-22807 (2013); doi: 10.1364/OE.21.022799

[7] J. Alfonso, et al, Design of an optical sensing interrogator using an edge filter scheme, Microwave \& Optoelectronics Conference 1, (2013); doi: 10.1109/IMOC.2013.6646509

[8] A. Li, et al, Design and Implementation of DualEdge Filter Demodulation System Based on the Dual LPFG and Embedded Technology, Instrumentation, Measurement, Computer, Communication and Control 1582, (2013); doi: 10.1109/IMCCC.2013.352 